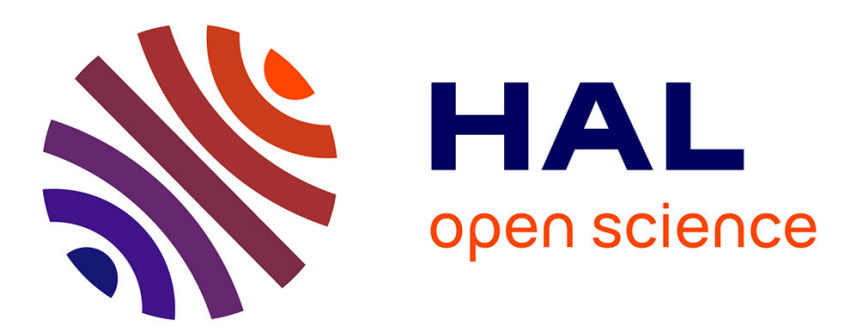

\title{
Characterization of flow contributions to drag and lift of a circular cylinder using a volume expression of the fluid force
}

L Fiabane, M Gohlke, Olivier Cadot

\section{- To cite this version:}

L Fiabane, M Gohlke, Olivier Cadot. Characterization of flow contributions to drag and lift of a circular cylinder using a volume expression of the fluid force. European Journal of Mechanics B/Fluids, 2011, 30 (3), pp.311-315. 10.1016/j.euromechflu.2010.12.001 . hal-01289951

HAL Id: hal-01289951

https://hal-ensta-paris.archives-ouvertes.fr/hal-01289951

Submitted on 17 Mar 2016

HAL is a multi-disciplinary open access archive for the deposit and dissemination of scientific research documents, whether they are published or not. The documents may come from teaching and research institutions in France or abroad, or from public or private research centers.
L'archive ouverte pluridisciplinaire HAL, est destinée au dépôt et à la diffusion de documents scientifiques de niveau recherche, publiés ou non, émanant des établissements d'enseignement et de recherche français ou étrangers, des laboratoires publics ou privés. 


\title{
Characterization of flow contributions to drag and lift of a circular cylinder using a volume expression of the fluid force.
}

\author{
L. Fiabane ${ }^{\mathrm{a}, \mathrm{b}, *}$, M. Gohlke ${ }^{\mathrm{b}}$, O. Cadot ${ }^{\mathrm{a}}$ \\ ${ }^{a}$ Unité de Mécanique, École Nationale Supérieure de Techniques Avancées ParisTech, \\ Chemin de la Hunière, 91761 Palaiseau Cedex, France \\ ${ }^{b}$ PSA Peugeot Citroën, Direction de la Recherche et de l'Ingénierie Avancée, Route de \\ Gisy, 78140 Vélizy Villacoublay, France.
}

\begin{abstract}
A 2D numerical simulation of the flow around a circular cylinder is investigated during the onset of unsteadiness within the range of Reynolds numbers between 50 and 400. Using the recent formulation of $\mathrm{Wu}, \mathrm{Lu}$ and Zhuang [J. Fluid Mech. 576, (2007)], the fluid force is successfully approximated by a volume integral of a force density over a small flow domain surrounding the cylinder. The domain does contain neither the detached vortices in the wake nor the vortex formation region. Using the vorticity laplacian, the domain is dynamically divided into two regions: the external flow region containing the two separated vortex layers and the back-flow region between these two vortex layers. The integration of the force density on either the separated vortex layers or the back-flow region gives two instantaneous contributions to the total force. For the mean drag it is found that the back-flow contribution increases from almost $0 \%$ of the total drag at $R e=50$ to $20 \%$ of the total drag at $R e=400$. The separated vortex layers contribution is found to decrease as $\left(a+b R e^{-1 / 2}\right)$. Concerning the force fluctuations, both regions contribute similarly to the lift oscillations, while only the back-flow region is responsible for the drag oscillations. This alternative comprehension of the fluid force origin is discussed and compared to that of the classical pressure/viscous formulation.
\end{abstract}

Keywords: Mathematical formula, Vortex dynamics, Separated flows, Drag

*Corresponding author: lionel.fiabane@ensta.org 
reduction

\section{Introduction}

The precise origin of the force in the flow, i.e. the exact phenomena or structures in the vicinity of a body that create the force is a subject of great importance, as well as from a fundamental point of view and for very practical applications. Although it is understood and agreed that what happens near a body directly creates the force, those are generally estimated through a pressure-viscous stress integration over the body surface as:

$$
\mathbf{F}(t)=\oint_{\mathcal{B}_{b o d y}}(-p \underline{\underline{\mathbf{I}}}+\underline{\underline{\boldsymbol{\tau}}}) \cdot \mathbf{n} d S
$$

where $\mathbf{F}(t)$ is the instantaneous aerodynamic force vector, $p$ is the pressure, $\mathbf{I}$ the identity matrix, $\boldsymbol{\tau}$ is the viscous stress tensor and $\mathbf{n}$ is the normal vector to the body surface. $\overline{\bar{T}}$ The projection of equation (1) on the flow direction $\mathbf{e}_{\mathbf{x}}$ separates the drag into two contributions:

$$
D_{p}=\oint_{\mathcal{B}_{b o d y}}(-p \underline{\underline{\mathbf{I}}} \cdot \mathbf{n}) \cdot \mathbf{e}_{\mathbf{x}} d S, \quad D_{f}=\oint_{\mathcal{B}_{b o d y}}(\underline{\underline{\boldsymbol{\tau}}} \cdot \mathbf{n}) \cdot \mathbf{e}_{\mathbf{x}} d S,
$$

with the corresponding force coefficient:

$$
C_{D p}=\frac{D_{p}}{\frac{1}{2} \rho U^{2} d l}, \quad C_{D f}=\frac{D_{f}}{\frac{1}{2} \rho U^{2} d l},
$$

where $U$ is the upstream uniform velocity, $d$ is the cylinder's diameter, $l$ its span (set equal to 1 in two dimensions) and $\rho$ the fluid density. $D_{p}$ depends on the pressure and takes into account the form drag and the induced drag. The friction drag $D_{f}$ depends on the viscous stress tensor and is known to evolve with the Reynolds number as $R e^{-1 / 2}$ [1] (defined as $R e=$ $\frac{U d}{\nu}$ where $\nu=\frac{\mu}{\rho}$ is the kinematic viscosity). The role of flow structures surrounding the cylinder could be investigated by studying their fingerprint at the body surface, however the non-locality of the pressure renders this approach difficult. In the case of bluff bodies, it can be shown [2] that the high drag is due to a strong base pressure induced by flow separation and vortex formation. These ingredients cannot be separated using equation (1).

The present study propose therefore to investigate these origins through a spatial decomposition of the flow made possible by the pioneering work of 
Wu et al. [3] (see also [4]), who introduced an alternative calculation free of pressure. This formulation of the aerodynamic force is established from a momentum budget equation and vectorial transformations. The expression reads in two dimensions:

$$
\begin{aligned}
\mathbf{F}(t)= & -\mu \int_{\mathcal{F}} \nabla^{2} \omega \mathbf{x}^{\perp} d V \\
& +\mu \oint_{\mathcal{B}}\left([(\hat{\mathbf{n}} \cdot \nabla) \omega] \mathbf{x}^{\perp}+\omega \hat{\mathbf{n}}^{\perp}\right) d S,
\end{aligned}
$$

where $\mu$ is the dynamic viscosity, $\omega$ the vorticity, $\mathbf{x}=x \mathbf{e}_{\mathbf{x}}+y \mathbf{e}_{\mathbf{y}}$ the position vector, $\mathbf{x}^{\perp}=y \mathbf{e}_{\mathbf{x}}-x \mathbf{e}_{\mathbf{y}}, \mathcal{F}$ the integration domain (or field), $\mathcal{B}$ the external boundary of this domain, $\hat{\mathbf{n}}=\hat{n}_{x} \mathbf{e}_{\mathbf{x}}+\hat{n}_{y} \mathbf{e}_{\mathbf{y}}$ the normal vector to this boundary pointing outside the domain and $\hat{\mathbf{n}}^{\perp}=\hat{n}_{y} \mathbf{e}_{\mathbf{x}}-\hat{n}_{x} \mathbf{e}_{\mathbf{y}}$. In the case of a circular cylinder at large $R e=9500, \mathrm{Wu}$ et al. [3] showed that the boundary integral (second term on the right handside of equation (4)) decays quickly as the integration domain $\mathcal{F}$ increases. Hence for a sufficiently large domain, the force can be expressed as a field expression only which allows a decomposition into different areas corresponding to flow structures. This is an advantage compared to other formulations that are also free of pressure but for which the boundary terms cannot be neglected (see for instance $[5,6]$ and the references therein). Wu et al. also showed that the integrand in the field integral is prominent in the vicinity of the cylinder. This means that the vortices, once shed, do not contribute to the fluid force. In addition to $\mathrm{Wu}$ et al.'s work, the present paper provides a spatial decomposition of the flow around the cylinder (back-flow region and external flow) and a characterization of their instantaneous contributions to the fluid force.

\section{Numerical simulation}

We choose to investigate equation (4) in the case of the solution of the 2D Navier-Stokes equations for the problem of a circular cylinder (shown in figure 1) in a uniform flow at moderate Reynolds numbers. In order to constitute a database for different Reynolds numbers ranging from 50 to 400 the numerical simulation is performed using a computational code based on a non-dimensional vorticity-streamfunction formulation. This kind of solver has been chosen for the accuracy of both the vorticity and its spatial derivatives which is crucial in the resolution of equation (4). Briefly, a coordinate 
transformation is used in order to treat the problem in a regular rectangular domain which enables the use of finite differences for all the spatial derivatives with a good accuracy. This is done thanks to a conformal mapping of the physical domain with a polar coordinate system $(r, \theta)$ into a rectangular domain $(X, Y)$ combined with an exponential spatial elongation function to make it regular. In this study the axes $(X, Y)$ of the rectangular computation domain represent in the physical domain the radius $r$ and the angle $\theta$ respectively. All the equations are solved in this calculation domain; its resolution is $500 \times 500$, the step in $\theta$ is $\frac{2 \pi}{500}$ and the radius goes from $0.5 d$ to $25 d$. The numerical method is the same as the one used and described in details in [7] except for the use of a physical domain of circular shape in the conformal mapping instead of a NACA shape. The same kind of study has been made using a commercial code based on finite volumes solving velocity and pressure. The results are equally good but the solver needs a more refined grid to obtain reliable spatial derivatives of the velocity.

\section{Results}

\subsection{Volume formulation of the fluid force}

The mean drag coefficient computed using equation (4) with a circular domain of diameter $5 d$ is shown in figure 3(a) as a function of $R e$. This mean drag coefficient is taken as a reference and simply refered as $C_{D}$ in the rest of the paper. In this figure we can see a very good agreement with Henderson's fit [8] also based on numerical simulations. We minimize the size of the domain $\mathcal{F}$ in order to obtain a value with an instantaneous error below $1 \%$ of the value of the reference force obtained from equation (4) with the $5 d$ circular domain. We choose rectangle domains, bounded vertically in $y$ by $\pm 0.95 d$ (fixed boundaries) and in $x$ by a fixed upstream limit at $-0.75 d$ and by a varying boundary downstream at $1 d$ to $0.75 d$ for $R e$ from 50 to 400. The integration domain $\mathcal{F}$ for $R e=300$ is displayed in figure 1 (a). For this Reynolds number the integrals due to the gradient of vorticity (second term on the right handside in equation (4)) calculated over each side of the $\mathcal{F}$ domain are much smaller than the total force, by an order of $10^{-4}$. The integrals over the fixed boundaries of the normal vorticity on the right handside in equation (4) are also smaller than the total force by the same order, and there is only one significant remaining term : the integral of the 

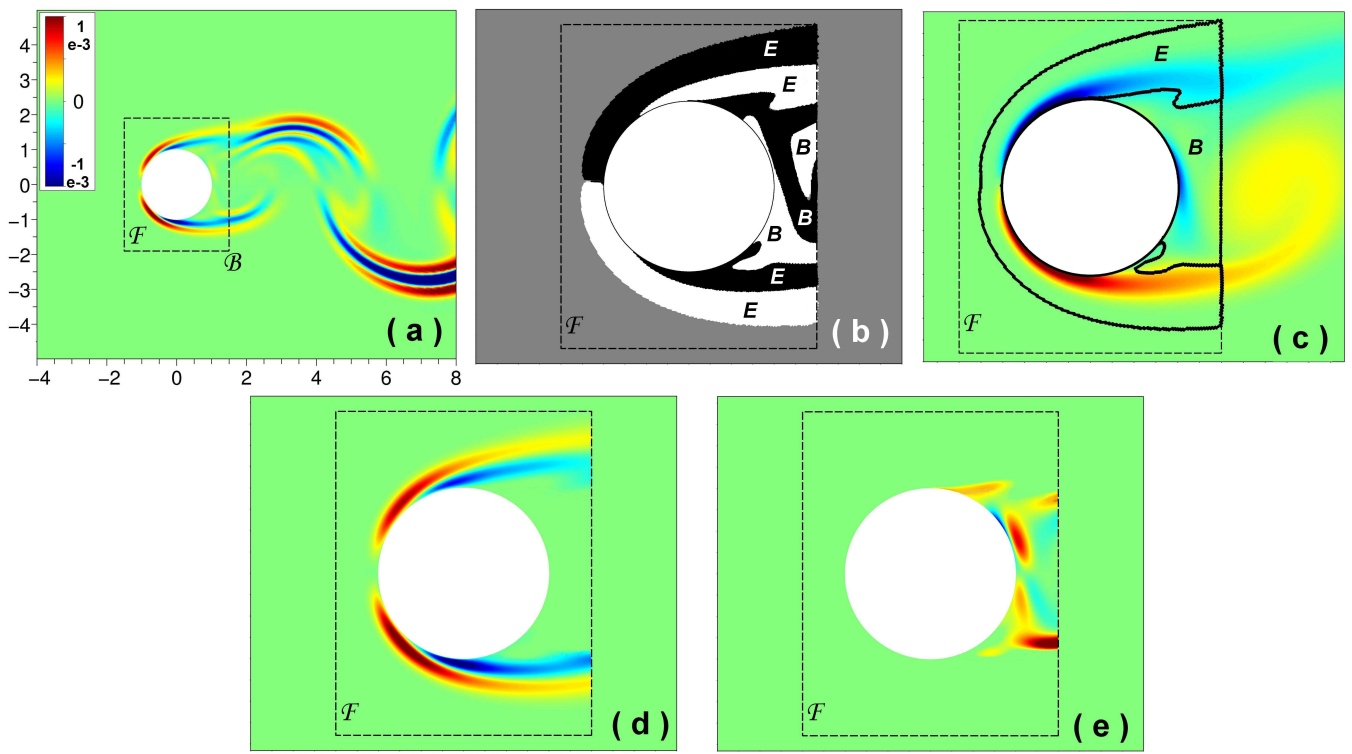

Figure 1: Instantaneous fields at $R e=300$, contributions to the drag force density of the external flow ' $E$ ' and the back-flow region ' $B$ ' defined in the integration domain $\mathcal{F}$ of external boundary $\mathcal{B}$ displayed by the dashed line ; (a) Drag force density field $\mathbf{f}_{\mathbf{V}} \cdot \mathbf{e}_{\mathbf{x}}$ as defined in equation (5); (b) Sign of $\nabla^{2} \omega$ (black: -1 , white : +1) with the different areas associated to the external flow ' $E$ ' or the back-flow region ' $B$ '; (c) Vorticity field, the continuous line shows the boundaries of the external flow ' $E$ ' and the back-flow region ' $B$ '; (d) Drag force density $\mathbf{f}_{\mathbf{V}} \cdot \mathbf{e}_{\mathbf{x}}$ for the external flow ' $E$ '; (e) Drag force density $\mathbf{f}_{\mathbf{V}} \cdot \mathbf{e}_{\mathbf{x}}$ for the back-flow region ' $B$ '. The scale in figure (e) is multiplied by a factor 5 compared to that of figure (d).

normal vorticity on the right handside in equation (4) calculated over the downstream boundary of the $\mathcal{F}$ domain. This last integral accounts for less than $1 \%$ of the total force and is therefore neglected, which is consistent with [3]. So, the force can thus be estimated instantaneously only by the field contribution only (right handside in equation (4)):

$$
\mathbf{F}(t) \approx \int_{\mathcal{F}} \mathbf{f}_{\mathbf{V}}(\mathbf{x}, t) d V=-\mu \int_{\mathcal{F}} \nabla^{2} \omega \mathbf{x}^{\perp} d V .
$$

\subsection{Flow decomposition}

It is worth noticing that the domain $\mathcal{F}$ does cover neither the wake (as mentioned previously and found in [3]) nor the vortex formation region. In figure 1(a), we can also see that the drag force density is concentrated in high positive (red) and high negative (blue) areas. From equation (5), these 
areas are intimately related to the vorticity distribution around the cylinder that is displayed for the same instant in figure 1(c). We can distinguish three different sources of vorticity production on the cylinder. The first two sources are created at the surface by both developments of boundary layers due to the oncoming flow on the front of the cylinder. Both boundary layers then separate from the wall and produce two shear layers of opposite signs. We will refer to these two structures as the vortex layers. The third source of vorticity appears at the rear of the cylinder and will be refered later as the back-flow region (in blue in figure 1(c)). This vorticity results from the interaction between the cylinder, the shear layers and the vortex roll-up that occurs downstream (see the yellow vortex in figure 1(c)).

The aim is now to segregate the flow into different parts. We therefore consider the vorticity laplacian $\nabla^{2} \omega$ which appears in equation (5). Areas of different signs of vorticity laplacian are separated using the sign function. The result is displayed in figure $1(\mathrm{~b})$. In the $\mathcal{F}$ domain we thus obtain seven areas which are combined to divide the flow into two parts. The first part constituted by areas denoted with letters ' $B$ ' is localized on the back-flow region (see figure $1(\mathrm{c})$ ) ; the second part associating ' $E$ ' areas is called the external flow and encompasses the vortex layers (see figure 1(c)). The two instantaneous regions ' $E$ ' and ' $B$ ' enclose totally the area of the integration domain $\mathcal{F}$ where the force density displayed in figure $1(\mathrm{a})$ is non zero. Figure 1(d) shows the drag force density field for the external flow only, whereas figure 1(e) shows the drag force density field for the back-flow region only. The same approach is used for the lift. Next we will analyse the contributions to the total drag $C_{D}$ of the regions ' $E$ ' and ' $B$ '.

\subsection{Drag and lift contributions}

Instantaneous contributions to the fluid force are obtained by integrating the force density over the corresponding regions ' $E$ ' and ' $B$ '. They are shown at $R e=300$ in figure 2. In the case of the drag coefficient (figure 2(a)) it is found that $90 \%$ of the total mean drag originates from the vortex layers in region ' $E$ ' while almost all the fluctuations are imposed by the back-flow region ' $B$ '. For the lift coefficient (figure $2(\mathrm{~b})$ ), we find that both the external 

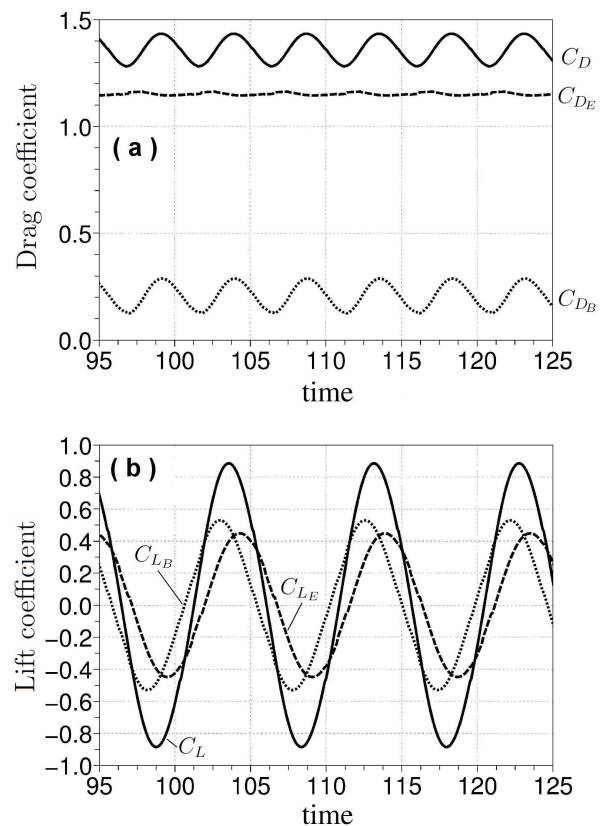

Figure 2: Instantaneous force as function of non-dimensional time for $R e=300$. Plain line is the sum of the contributions, dashed line is the external flow contribution, and dotted line is the back-flow contribution. (a) Drag coefficients. (b) Lift coefficients. 

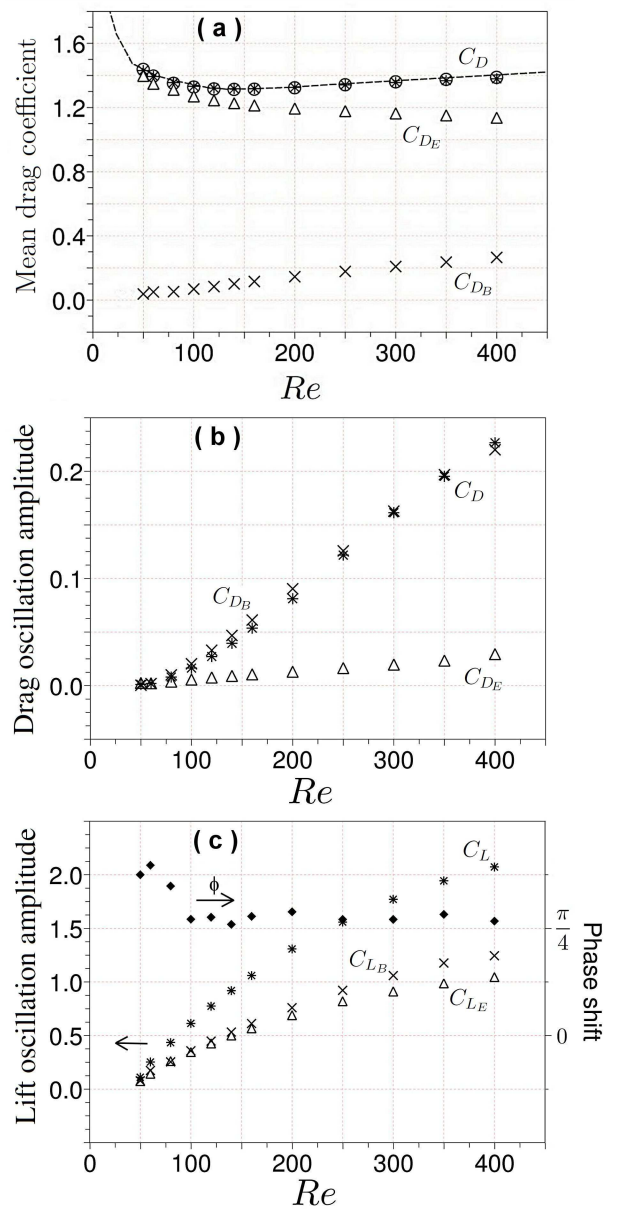

Figure 3: (a) Mean drag coefficient of the cylinder estimated from the density force in the reference domain $5 d(\circ)$, in $\mathcal{F}$ domain $(*)$, the dashed line is the fit from [8]. $C_{D_{E}}(\triangle)$ is the external flow contribution. $C_{D_{B}}(\times)$ is the back-flow region contribution ; (b) Drag oscillation amplitudes as function of the Reynolds number for the external flow $(\triangle)$, the back-flow region $(\times)$ and the total force $(*)$; (c) Lift oscillation amplitudes (left axis) as function of the Reynolds number for the external flow $(\triangle)$, the back-flow region $(\times)$ and the total force $(*)$. $(\checkmark)$ denotes the phase shift $\phi$ between the external and the back- flow lift (right axis). 
flow and the back-flow region contribute similarly. We also observe that the back-flow region lift is phase advanced compared to that of the external flow lift.

The flow separation into the external flow and the back-flow regions is successfully performed for $50 \leq R e \leq 400$. For each Reynolds number, we compute the mean drag coefficients (the mean lift is zero due to the symmetry of the flow around the cylinder) as well as the lift and drag oscillation amplitudes. The mean drag coefficients are plotted as a function of the Reynolds number in figure $3(\mathrm{a})$, with triangles $(\triangle)$ for the external flow drag $C_{D_{E}}$ and with crosses $(\times)$ for the back-flow $\operatorname{drag} C_{D_{B}}$. As observed previously, most of the drag is due to the vortex layers in the external flow. At $R e=50$ their contribution is almost equal to the total drag $C_{D}$; then it decreases continuously as the Reynolds number increases. Simultaneously the back-flow drag rises. Figure 3(b) shows that the drag oscillation amplitudes are only due to the back-flow region in the considered range of Reynolds numbers, while the lift oscillation amplitudes for both the external and the back-flow remain equivalent (figure 3(c)). The phase shift $\phi$ between the external flow lift and the back-flow lift remains fairly constant $\left(\phi \approx \frac{\pi}{4}\right)$ for $R e \geq 100$. For Reynolds number larger than 400, the flow becomes too complex in the back-flow region, making the association of the flow areas given by the sign of the vorticity laplacian into regions ' $E$ ' and ' $B$ ' impossible.

\section{Discussion}

We have so far determined the force contributions of two parts of the flow : the external flow and the back-flow region. The force density in the external flow is produced by the vortex layers. The force density in the back-flow region is related to the vorticity created at the rear of the cylinder (figure 1(c)). We discuss now the evolution of these contributions.

The vortex layers contribution is found to evolve as $C_{D_{E}}=C_{0}+2.75 R e^{-1 / 2}$ (figure 4(a)). The vortex layers create locally a friction drag at the surface of the cylinder before the separation. This is consistent with the observed dependence with the Reynolds number [2]. Moreover the external drag $C_{D_{E}}$ 

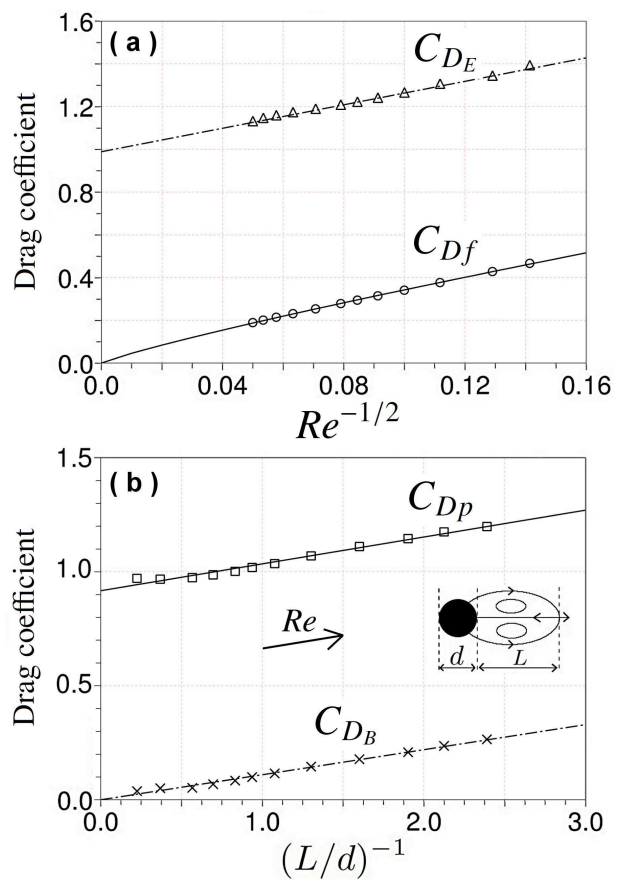

Figure 4: (a) External flow drag $C_{D_{E}}$ and friction drag $C_{D f}$ dependence with $R e$; (b) Back-flow drag $C_{D_{B}}$ and form drag $C_{D p}$ dependence with $(L / d)$. 
also contains a large constant contribution which must be ascribed to the separation of the boundary layers from the cylinder.

We consider next the back-flow drag $C_{D_{B}}$. It is found to increase with the Reynolds number (figure 3(b)) to reach a contribution of about $20 \%$ of the total drag $C_{D}$ at $R e=400$. This evolution is due to an increase of the force density in the back-flow region that is created by the interactions between the shear layers, the vortex roll-up and the cylinder. This intensification of the vorticity interactions is also accompanied by the increase of the drag fluctuations in the back-flow region (figure 3(b)) that are correlated to the vortex roll-up dynamics: the larger the Reynolds number, the closer to the base of the cylinder the roll-up [9] and the stronger the drag fluctuations. The increase of the fluctuations with the Reynolds number can also be observed in the case of the lift (figure 3(c)) for which the roll-up in the backflow region drives the whole process [10], as expressed by the phase advance observed between the back-flow lift and the external lift (we have however no explanation at the moment to why the phase-shift becomes constant for $R e \geq 100)$. A way to estimate the distance between the cylinder base and the vortex formation is to compute the recirculation bubble length $L$ [11], defined from the downstream stagnation point of the dividing streamline of the mean flow (see sketch in figure 4(b)). The relationship between $C_{D_{B}}$ and $L$ is found to be almost linear with $C_{D_{B}}=0.11(L / d)^{-1}$ (figure $4(\mathrm{~b})$ ). This means that as the Reynolds number increases, the roll-up occurring closer to the cylinder creates stronger interactions between the rear vorticity and the shear layers, resulting in an increased back-flow drag.

It is interesting to relate the present results to what can be achieved in terms of passive control of bluff body wakes. A splitter plate [12] or a small control cylinder $[13,14,15]$ in the wake are known to reduce drag and suppress force fluctuations. The largest drag reduction that can be achieved is about 30\% (with no reattachment and when the separation points are not significantly affected). It has been shown [16] that this control technique have the property to push further downstream the vortex roll-up and then to increase the recirculation bubble length. In the light of the present study, these techniques suppress the back-flow region drag $C_{D_{B}}$ and its fluctuations as the vortex roll-up is too far away from the body to induce rear vorticity.

We can finally compare our present flow decomposition with the pressure and the viscous stress contributions. We have computed the form $\operatorname{drag} C_{D p}$ 
(since the flow is two-dimensional, the induced drag is zero) and the viscous shear drag $C_{D f}$ (figure 4). Our fit for viscous shear drag in figure 4(a) gives $C_{D f}=2.54 R e^{-0.44}$ (similar to that of [8]) which is very close to the dependence in $R e$ that we found for the external flow drag. Hence the vortex layers capture the viscous drag. The fit for pressure drag in figure 4(b) gives $C_{D p}=C_{0}{ }^{\prime}+0.12(L / d)^{-1}$, which is very close to the dependence in $L$ we found for the back-flow drag. The form drag is thus captured by two structures: the vortex layers through a constant contribution $C_{0}$ in $C_{D_{E}}$ that depends on the separation of the shear layers from the cylinder, and the vorticity contained in the back-flow region induced by the vortex roll-up dynamics.

In conclusion, we can say that we have studied a new way to characterize some flow contributions to the fluid force. For the circular cylinder under the laminar vortex shedding stage, the force density formulation offers a quantitative decomposition of the mean drag origin. This promising method offers an alternative comprehension of the force origin to the classical pressure/viscous stress decomposition. In future work, we plan to apply this method to complex flows around controlled 2D bluff bodies [15] and 3D bluff bodies [17] to get new insights into force-flow structures relationship. The challenge is then twofold: fundamentally it relies on a proper identification of the structures, and numerically it requires to compute the vorticity laplacian accurately.

The authors are gratefull to Olivier Le Maitre for providing the numerical code and to Maurice Rossi and Romain Pennel for their critical readings of the paper.

\section{References}

[1] G.K. Batchelor, An Introduction to Fluid Dynamics, Cambridge University Press, 2002.

[2] A. Roshko, Perspectives on bluff body aerodynamics. J. Wind Eng. Ind. Aerodyn. 49 (1993) 79-100.

[3] J.-Z. Wu, X.-Y. Lu, L.-X. Zhuang, Integral force acting on a body due to local flow structures. J. Fluid Mech. 576 (2007) 265-286. 
[4] J.-Z. Wu, H.-Y. Ma, M.-D. Zhou, Vorticity and Vortex Dynamics, Springer, 2006.

[5] F. Noca, D. Shiels, D. Jeon, Measuring instantaneous fluid dynamic forces on bodies, using only velocity fields and their derivatives. J. Fluids Struct. 11 (1997) 345-350.

[6] L. Quartapelle, M. Napolitano, Force and Moment in Incompressible Flows. AIAA 21 (1983) 911-913.

[7] O.P. Le Maître, R.H. Scanlan, O.M. Knio, Estimation of the flutter derivatives of an NACA airfoil by means of Navier-Stokes simulation. $J$. Fluids Struct. 17 (2003) 1-28.

[8] R.D. Henderson, Details of the drag curve near the onset of vortex shedding. Phys. Fluids 7 , 9 (1995).

[9] M. Nishioka, H. Sato, Mechanism of determination of the shedding frequency of vortices behind a cylinder at low Reynolds number. J. Fluid Mech. 89 (1978) 49-60.

[10] J.-M. Chomaz, Global instabilities in spatially developping flows: nonnormality and nonlinearity. Ann. Rev. Fluid Mech. 37 (2005) 357-392.

[11] J.H. Gerrard, The mechanics of the formation region of vortices behind bluff bodies. J. Fluid Mech. 25 (1966) 401-413.

[12] C.J. Apelt, G.S. West, The effects of wake splitter plates on bluff-body flow in the range $10^{4}<R<5.10^{4}$. Part 2 J. Fluid. Mech. 71 (1975) 145-160.

[13] P.J. Strykowski, K.R. Sreenivasan, The control of Transittional Flows. AIAA Shear Flow Control Conference, March 1994, Paper 85-0559.

[14] C. Dalton, Y. Xu, J.C. Owen, The suppression of lift on a circular cylinder due to vortex shedding at moderate Reynolds numbers. J. Fluids Struc. 15 (2001) 617-628.

[15] V. Parezanovic, O. Cadot, The impact of a local perturbation on global properties of a turbulent wake. Phys. Fluids 21,7 (2009). 
[16] O. Cadot, B. Thiria, J.-F. Beaudoin, Passive Drag Control of a Turbulent Wake by Local Disturbances. IUTAM Symposium on Unsteady Separated Flows and their Control (June 2007), IUTAM Bookseries 14 (2009) 529-537.

[17] M. Gohlke, J.-F. Beaudoin, M. Amielh, F. Anselmet, Thorough analysis of vortical structures in the flow around a yawed bluff body. J. Turbulence $\mathbf{9 , 1 5}$ (2008). 